\title{
LE DROIT AU SERVICE DES PERSONNES DÉFAVORISÉES ?
}

Les effets pervers de la mise en œuvre du droit au logement opposable Pierre-Edouard Weill

Presses de Sciences Po (P.F.N.S.P.) | « Gouvernement et action publique »

$2013 / 2 n^{\circ} 2$ | pages 279 à 302

ISSN 2260-0965

ISBN 9782724632958

Article disponible en ligne à l'adresse :

http://www.cairn.info/revue-gouvernement-et-action-publique-2013-2-page-279.htm

\section{Pour citer cet article :}

Pierre-Edouard Weill, « Le droit au service des personnes défavorisées ? Les effets pervers de la mise en œuvre du droit au logement opposable », Gouvernement et action publique 2013/2 ( $\left.{ }^{\circ} 2\right)$, p. 279-302.

DOI 10.3917/gap.132.0279

Distribution électronique Cairn.info pour Presses de Sciences Po (P.F.N.S.P.).

(C) Presses de Sciences Po (P.F.N.S.P.). Tous droits réservés pour tous pays.

La reproduction ou représentation de cet article, notamment par photocopie, n'est autorisée que dans les limites des conditions générales d'utilisation du site ou, le cas échéant, des conditions générales de la licence souscrite par votre établissement. Toute autre reproduction ou représentation, en tout ou partie, sous quelque forme et de quelque manière que ce soit, est interdite sauf accord préalable et écrit de l'éditeur, en dehors des cas prévus par la législation en vigueur en France. Il est précisé que son stockage dans une base de données est également interdit. 


\title{
LE DROIT AU SERVICE DES PERSONNES DÉFAVORISÉES ?
}

\author{
Les effets pervers de la mise en œuvre du droit \\ au logement opposable ${ }^{1}$
}

Pierre Édouard Weill

Résumé : Cet article interroge les conditions de mise en œuvre du droit des personnes défavorisées à travers l'analyse du processus d'offre de logement au titre du droit au logement opposable (DALO). Derrière une étiquette juridique et homogénéisante, on discerne des profils spécifiques de ménages "prioritaires " au titre du DALO, dont les caractéristiques sociales déterminent les propositions de logement ou d'hébergement qui leur sont faites, tout comme leur réception. On explique ainsi comment ceux qui parviennent à faire valoir leur droit sont majoritairement orientés vers des " quartiers sensibles ", lorsqu'ils ne doivent pas se contenter d'une place d'hébergement, mais aussi pourquoi ces propositions entrainent leur satisfaction, leur résignation ou leur refus. Cet article montre finalement les limites et les effets pervers d'une tentative improbable d'optimisation de la politique du logement des personnes défavorisées par le recours à la justice administrative, qui aboutit à la fois au ciblage accru de l'attribution des logements sociaux et à une concentration renforcée de la pauvreté.

MOTS-CLÉS : ACTION PUBLIQUE - JUSTICE - LOGEMENT - OPTIMISATION - RESSORTISSANTS

\section{IS LAW EVER USEFUL FOR DISADVANTAGED PEOPLE? THE UNINTENDED EFFECTS OF THE IMPLEMENTATION OF THE ENFORCEABLE RIGHT TOHOU- SING IN FRANCE}

Abstract: This article analyzes the implementation gap of social rights for disadvantaged people by focusing on the processing of priority applicants for the enforceable right to housing (DALO) in France. Behind the homogenizing legal label of DALO, distinctions must be made between different types of applicants, whose social characteristics and trajectories determine both the type of accommodation proposed by the local officials and their contrasted expectations in terms of housing. This explains why accommodations proposed to homeless people who manage to assert their rights are predominantly located in the poorest areas, when they are not simply directed to emergency shelters. Depending on their backgrounds and expectations they may be satisfied, resigned or refuse the offer. Lastly, this paper shows the limitations and the unintended effects of

1. Je remercie Fabien Desage, Vincent Dubois et Jay Rowell pour leurs lectures de versions antérieures de ce texte, ainsi que les évaluateurs anonymes de la revue pour leurs précieux conseils. Un grand merci également à Benjamin Soulet pour la réalisation des cartes qui illustrent cet article. 
the effort to "optimize" social housing policy through the individual recourse to justice, as it increasingly targets policy on the most disadvantaged people and contributes to the concentration of poverty in certain urban areas.

\section{KEYWORDS: HOUSING POLICY - IMPLEMENTATION - JUSTICE - URBAN SEGREGATION}

Depuis son entrée en vigueur le $1^{\mathrm{er}}$ janvier 2008, la loi instituant le droit au logement opposable, dite «DALO $»^{2}$, introduit une possibilité de recours à la justice administrative en vue d'obtenir un logement adapté. Suite à un recours préalable auprès d'une commission de médiation départementale composée de représentants de l'État, des collectivités locales, des bailleurs sociaux et privés, ainsi que des associations œuvrant pour l'accès au droit ou le logement des personnes défavorisées, les requérants peuvent voir la situation de leur ménage classée "prioritaire et urgente " sous certaines conditions ${ }^{3}$. Lorsque le recours au DALO n'est pas rejeté, ce qui se produit le plus souvent ${ }^{4}$, les agents de l'État doivent faire en sorte qu'une proposition soit formulée. Si tel n'est pas le cas dans les 6 mois suivant la décision de la commission, le juge administratif peut sanctionner le préfet pour son incapacité à assurer l'obligation de résultat qui incombe à l'État.

La reconnaissance d'un tel « droit-créance médiateur » - le droit n'étant pas tant considéré pour lui-même que comme une médiation pour atteindre un objectif d'intégration des plus défavorisés (Lafore, 1998) - apparaît a priori comme un facteur de progrès social. Elle permet théoriquement à la demande des «sans-abris » et des «mal logés » de s'exprimer dans la «forme par excellence du discours légitime » (Bourdieu, 1986, p. 15) et de produire des connaissances sur les besoins en termes de logement très social, tout en y apportant une réponse. La mise en œuvre du DALO donne ainsi à voir une forme de «redéfinition du judiciable» annoncée par Michel Foucault (1977) lors d'un séminaire du Syndicat de la

2. Loi $n^{\circ}$ 2007-290 du 5 mars 2007 instituant le droit au logement opposable et autres mesures de cohésion sociale.

3. Les ménages éligibles sont de nationalité française ou disposent d'un titre de séjour en cours de validité et répondent aux conditions d'accès à un logement social et leur situation est reconnue "prioritaire " si elle correspond à un ou plusieurs des 6 critères suivants : être sans domicile ; être menacé d'expulsion sans relogement, soit hébergé dans une structure d'hébergement ou une résidence hôtelière à vocation sociale de façon continue depuis plus de 6 mois ou logé temporairement dans un logement de transition depuis plus de 18 mois ; être logé dans des locaux impropres à l'habitation ou présentant un caractère insalubre ; être logé dans un logement ne présentant pas d'éléments d'équipement et de confort exigés (absence de chauffage, d'eau potable, etc...) à condition d'avoir à sa charge au moins un enfant mineur ou une personne handicapée ou de présenter soi-même un handicap; être logé dans un logement présentant une surface habitable au plus égale à $16 \mathrm{~m}^{2}$ pour un ménage sans enfant ou 2 personnes, augmentée de $9 \mathrm{~m}^{2}$ par personne en plus, dans la limite de $70 \mathrm{~m}^{2}$ pour 8 personnes et plus, à condition d'avoir à sa charge au moins un enfant mineur, une personne handicapée ou de présenter soi-même un handicap ; être demandeur d'un logement social depuis un délai anormalement long (qui varie d'un département à l'autre) sans avoir reçu de proposition adaptée.

4. Selon les données nationales disponibles au $1^{\text {er }}$ janvier 2008, le nombre de recours dépasse les 6000 par mois et il s'élève à 295313 fin juin 2012. Le taux de validation des recours est en baisse continue : il passe de 44,2 \% entre mars 2008 et décembre 2010, à 38,9\% entre janvier 2010 et décembre 2011, puis à $37 \%$ au premier semestre 2012 (Comité de suivi de la mise en œuvre de la loi DALO, 2012). Catherine Bourgeois s'est intéressée plus précisément au classement des recours en commissions de médiation départementales DALO (Bourgeois, 2012). 
Magistrature ${ }^{5}$. Comme d'autres domaines de la vie sociale, le logement des personnes défavorisées est "pris en charge, traversé par une instance de type judiciaire ». Le rôle du juge, comme des profanes qui siègent en commission de médiation, est de "définir non pas tellement ce qui est légal et ce qui est illégal », mais plutôt « un optimum fonctionnel pour le corps social » (Foucault, 1977).

Cet article envisage donc le DALO comme une improbable tentative d'" optimisation " de la politique du logement social par le recours à la justice administrative. Une sociologie de l'action publique attentive aux acteurs en charge de sa mise en œuvre, comme aux administrés, met en valeur ses limites et ses effets pervers. Tandis que de nombreux individus n'ont pas recours au DALO, d'autres ne parviennent pas à faire valoir leur droit, bien que leur situation soit "prioritaire et urgente " au regard de la loi. L'application du DALO tend à exclure les plus éloignés des institutions et les moins conformes aux attentes des acteurs du logement des personnes défavorisées, qui sont aussi souvent les plus démunis (Weill, 2012). Sa mise en œuvre est d'autant moins synonyme d'effectivité de la règle de droit (Lascoumes, Serverin, 1986), que les ménages «prioritaires » ne se voient pas systématiquement proposer de logement, même après la condamnation du préfet par le juge administratiff ${ }^{6}$. Si l'enquête se focalise ici sur les publics faisant l'objet d'une offre de la part des acteurs du logement des personnes défavorisées, elle montre à quel point l'« exigence d'abstraction universalisante » du droit (Sabbagh, 2003, p. 309) et l'étiquette homogénéisante apposée sur les «ménages DALO » masquent la diversité des « ressortissants " de l'action publique (Warin, 1999) et les divergences des acteurs institutionnels qui la mettent en œuvre.

En témoignent d'une part les refus des bailleurs sociaux de loger les ménages " prioritaires » les plus démunis, par souci de préserver les franges les plus attractives de leur parc. Lorsque les ménages " prioritaires " ne sont pas orientés vers une structure d'hébergement, ils se voient majoritairement proposer d'être logés dans les quartiers de grands ensembles d'habitat social des zones les plus pauvres des grandes agglomérations. En témoignent d'autre part les refus d'offres de logement et d'hébergement des «sans-abris » ou de «mal logés ", qui, en dépit de leur situation, se révèlent insatisfaits de l'action publique menée en leur faveur. C'est particulièrement le cas lorsqu'ils sont réorientés vers une structure d'hébergement. Cela l'est aussi lorsque les logements proposés se situent dans les quartiers

5. Ce séminaire se tient au Château de Goutelas, site aménagé par Paul Bouchet, l'un des animateurs du mouvement "Critique du droit» (Israël, 2009). C'est en tant que conseiller d'État honoraire, président de l'association ATD-Quart-Monde et membre du Haut Comité pour le Logement des Personnes Défavorisées qu'il se révèle, 25 ans plus tard, le principal acteur de la genèse du DALO (Houard, 2012).

6. L'essentiel du retard de l'offre de logement est situé dans les régions d'île-de-France et de Provence-AlpesCôte d'Azur, ainsi que dans les DOM-TOM. Le cas de la ville de Paris est particulier puisque la Capitale concentre $64 \%$ des ménages dont la situation est "prioritaire et urgente " n'ayant toujours pas bénéficié d'une offre de logement, alors qu'elle ne représente que $19 \%$ du total des recours (Comité de Suivi de la Mise en CEuvre du DALO, 2011). Dans les autres régions, y compris parmi les plus urbanisées, comme le Rhône-Alpes ou l'Alsace, la quasi-totalité des « ménages DALO » reçoit une proposition de logement. Selon les sources disponibles auprès du Haut comité pour le logement des personnes défavorisées au 30 juin 2012, environ $65 \%$ des ménages dont la situation a été déclarée " prioritaire et urgente " en commission ont reçu une proposition de logement au titre du DALO, estimation qui pourrait être légèrement rehaussée par le fait que, dans certains départements, des ménages sont relogés avant même que la commission n'ait eu le temps de statuer. 
paupérisés et stigmatisés, qui ne correspondent pas toujours aux aspirations résidentielles de ceux qui s'y voient assigner.

Le "peuplement " - entendu comme l'action publique sur la répartition spatiale des habitants (Morel Journel, Sala Pala, 2011) - est envisagé ici à travers le rôle des acteurs institutionnels et les appropriations plus ordinaires de leurs ressortissants. Certains chercheurs se sont intéressés aux systèmes d'attribution de logements sociaux, dont ils font ressortir les mécanismes de "discrimination informelle " (Tissot, 2005) affectant des populations considérées comme "à risques » (Simon, 2003). Leurs travaux se focalisent toutefois sur les acteurs institutionnels des politiques publiques, plutôt que sur leurs ressortissants. Les publics de l'hébergement d'urgence font d'ailleurs l'objet de travaux plus approfondis (Damon, 2002 ; Bruneteaux, 2007) que les demandeurs de logements sociaux, qui n'apparaissent souvent que sous la forme de collectifs politiquement mobilisés (Péchu, 2006). L'enquête de Dietrich-Ragon fait bien ressortir les attitudes des «mal logés » face aux politiques de lutte contre l'insalubrité (2011), et celle de Bacqué, Fijalkow et Vermeersch, 2010 analyse bien les comportements d'habitants d'immeubles conventionnés qui « deviennent HLM » (2010), mais ces enquêtes se focalisent sur le relogement de ménages modestes dans des «beaux quartiers " parisiens. À l'inverse, les travaux sur la ségrégation urbaine (Préteceille, 2006), les « effets de quartier » (Wilson, 1997, ou la «mauvaise réputation » des grands ensembles d'habitat social (Van San, 2004 ne s'intéressent pas véritablement au rapport des individus concernés à l'action publique.

Tout en s'inscrivant dans la continuité de ces travaux, l'analyse porte à la fois sur l'action publique et la manière dont les administrés s'en saisissent. Elle s'inscrit aussi à rebours d'une explication individualiste de ses effets pervers. L'offre de mobilité résidentielle aux bénéficiaires du DALO, comme son acceptation ou son refus, trouvent en effet leur explication au carrefour de logiques d'acteurs et de déterminants structurels (Grafmeyer, Authier, 2008). C'est ce que fait apparaître le traitement d'un matériau empirique diversifié, combinant les méthodes ethnographique et statistique. L'observation des pratiques des acteurs institutionnels, comme des requérants DALO, permet de saisir leurs motivations respectives. Elle favorise aussi le repérage des variables significatives dans les explications causales offertes par la mobilisation de techniques statistiques (Belorgey, 2011). En l'occurrence, l'analyse des correspondances multiples (ACM) rapporte d'abord systématiquement l'offre de logement ou d'hébergement proposée par les acteurs institutionnels aux données disponibles sur les «ménages DALO ». La méthode de classification ascendante hiérarchique $(\mathrm{CAH})^{7}$ distingue ensuite des types de ménages qui partagent des caractéristiques proches, se voient proposer les mêmes solutions en matière d'habitat et adoptent une semblable attitude face à ces dernières.

7. Ces deux techniques, couramment combinées, permettent à la fois de différencier des individus statistiques et de dégager des facteurs qui synthétisent les corrélations de l'ensemble des variables qualitatives qui les caractérisent. La première permet plutôt de montrer comment se polarisent les différentes modalités de variables caractéristiques des individus sur un plan factoriel, tandis que la seconde offre de répartir ces individus en groupes qui partagent le plus de caractéristiques communes en fonction de ces variables (Greenacre, Blasius, 2006). 


\section{- L'enquête}

Les résultats de cet article s'appuient sur une partie des matériaux d'une enquête finalisée sur plusieurs départements (Paris, Yvelines, Bas-Rhin, Vosges), dans laquelle plusieurs types de sources sont mobilisées : littérature grise (archives institutionnelles, rapports, publications institutionnelles et spécialisées), débats parlementaires, articles de presse nationale et locale ; réalisation d'entretiens auprès d'acteurs institutionnels [ $=103]$ ) ; observation de réunions publiques d'acteurs du logement, de réunions des commissions de médiation du Bas-Rhin [ $n=2]$, des Vosges [ $n=1]$, de Paris $[n=2]$, des Yvelines [ $n=2]$ et d'audiences de tribunaux administratifs $[n=6]$; observation ethnographique du travail administratif en préfecture, participation observante au travail d'accompagnement juridique en milieu associatif [sur un an et demi] et entretiens semidirectifs avec des requérants DALO [ $n=20]$; traitement statistique d'un échantillon de dossiers d'instruction de recours devant les commissions de médiation DALO de Paris, Versailles et Strasbourg [ $n=432]$.

Cependant, l'analyse porte tout particulièrement sur l'étude du cas de la Communauté Urbaine de Strasbourg (CUS), comparable à celui de la majorité des agglomérations de plus de 450000 habitants dans lesquelles le marché locatif est souvent relativement tendu. En plus des différents types de matériaux précédemment évoqués, des données précises sur la caractérisation sociodémographique du territoire de l'agglomération et la répartition socio-spatiale de l'offre de logement et d'hébergement aux "ménages DALO » ont été recueillies sur ce site. Une extraction du fichier de relogements DALO $[\mathrm{n}=562]$ de la Direction Départementale de la Cohésion Sociale (DDCS) du Bas-Rhin, entre 2009 et 2010, a notamment fait l'objet de différents traitements. Un échantillon de ménages [ $\mathrm{n}=384]$, tous caractérisés par leur composition familiale, leur adresse d'origine et une offre de logement ou d'hébergement a tout d'abord été constitué. La répartition de cette offre a ensuite été localisée sur deux cartes de la CUS. Ce territoire est caractérisé par un marquage des zones ciblées par les " politiques de la ville ", puis par les niveaux de revenu des ménages par " Îlots Regroupés pour l'Information Statistique » (IRIS), qui font référence à la taille visée de 2000 habitants par maille élémentaire (données INSEE 2007). Une analyse des correspondances multiples a par ailleurs été réalisée sur la base de l'échantillon de ménages précédent. Le refus ou l'acceptation de l'offre de logement ou d'hébergement ont finalement été intégrés à l'analyse, ce qui a donné lieu à la réalisation d'une classification ascendante hiérarchique.

La première partie de l'article met en valeur les apories de la répartition de l'offre d'habitat aux demandeurs de logement dont la situation est déclarée " prioritaire et urgente " au titre du DALO. Elle montre que les préventions des bailleurs à l'encontre des ménages dont les caractéristiques les font apparaître comme "à risques " renforcent paradoxalement la concentration de la pauvreté. La seconde partie s'attache quant à elle à discerner la pluralité des ménages des requérants qui parviennent à faire valoir leur droit. Elle analyse les appropriations socialement différenciées de l'action publique, qui se traduisent notamment par l'acceptation ou le refus de l'offre d'habitat proposée. 


\section{Les apories de la répartition de l'offre d'habitat}

Lorsqu'ils ne sont pas réorientés vers une structure d'hébergement, les ménages prioritaires se voient le plus souvent proposer un logement dans les quartiers ciblés par la politique de la ville. Cette offre n'est toutefois pas totalement homogène, de même que les caractéristiques et les situations en matière d'habitat des ménages « prioritaires ». Ceci étant, il s'agit de mettre ces éléments en rapport, afin de saisir comment les logiques de la répartition socio-spatiale de l'offre d'habitat favorisent la concentration des «bénéficiaires " du DALO dans les zones les plus pauvres. Bien que ce risque soit partiellement envisagé par les pouvoirs publics, les réticences de certains bailleurs à accueillir des ménages défavorisés limitent sa réduction.

\section{Un risque de concentration de la pauvreté mis en forme dans les débats nationaux}

De nombreux travaux s'intéressent aux phénomènes de gentrification des quartiers populaires liés aux politiques de régénération urbaine. Plus rares sont ceux qui mettent à jour des processus de reconcentration de la pauvreté (Lelévrier, 2010). Le rapport annuel du Haut Comité à l'Intégration $(\mathrm{HCl})$ de Blandine Kriegel dénonce cependant dès janvier 2008 les effets pervers du relogement des ménages prioritaires au titre du DALO sur le peuplement des "quartiers sensibles », accusant notamment la mise en œuvre du DALO de "contribuer à défavoriser l'intégration de certaines familles immigrées " ${ }^{8}$. Des élus de communes populaires de droite comme de gauche, dont les appartenances institutionnelles prennent souvent le pas sur les attaches partisanes (Gaxie, 1997), refusent quant à eux de "recevoir toute la misère du monde ${ }^{9}$ ". Ils critiquent tour à tour "une politique qui impose des arbitrages avec la réalisation de projets engagés ${ }^{10}$ " et "remet en cause le travail de terrain des collectivités territoriales " ainsi que leurs "stratégies fines de peuplement ${ }^{11}$ ". Ces élus voient leurs complaintes relayées jusqu'au sein du gouvernement de l'époque. La secrétaire d'État à la Ville dénonce ainsi des « risques de ghettoïsation ${ }^{12}$. Membre d'un gouvernement de la même couleur politique que celui à l'origine de la création du DALO, Alain Juppé condamne pourtant « une loi d'affichage, électoraliste et sans utilité ${ }^{13}$. Face aux remises en cause de l'application de la loi qui émanent des débats politique nationaux, les agents de l'État local oscillent entre inquiétudes, comme à Strasbourg et dans les Yvelines, et relativisme, comme à Paris, où le président de la commission de médiation affirme que «le problème de mixité sociale ne se pose pas véritablement » :

8. Rapport annuel du Haut Comité à l'Intégration (HCl) cité par Le Figaro du 21 janvier 2008.

9. Rodolphe Thomas, maire d'Hérouville-St-Clair, MODEM, $4^{\ominus}$ bilan parlementaire sur le DALO, Table ronde sur les engagements des partis politiques sur le DALO à la veille de l'élection présidentielle, Assemblée nationale, 30 novembre 2011.

10. Entretien avec Dominique Braye, député des Yvelines, Président du PUCA et rapporteur de la Loi DALO du 5 mars 2007, février 2010.

11. Intervention de François Puponni, député-maire de Sarcelles, Assemblée nationale, Séance en hémicycle du 9 février 2009.

12. Fadela Amara, Audition par la commission des affaires économiques de l'Assemblée, 9 septembre 2010.

13. Alain Juppé, Conseil municipal de Bordeaux, 26 septembre 2011. 
"Sur la capitale, la population est particulièrement diversifiée, même dans les arrondissements les plus populaires comme le $\mathrm{xIXe} !^{14}{ }^{\prime}$.

Les réticences à l'application du DALO liées aux accusations de concentration des pauvres dans les zones ciblées par la politique de la ville, sont cependant de mieux en mieux anticipées par l'administration centrale. Des statistiques sur le taux de logement en ZUS des ménages prioritaires ont été commandées, et une circulaire ministérielle a été adressée aux agents préfectoraux, leur commandant de faire en sorte que ce taux ne dépasse pas $30 \%$ à l'échelle du département ${ }^{15}$. Cet objectif apparaît néanmoins difficile à tenir dans les zones de forte tension du marché locatif, qui concentrent le plus grand nombre de recours et où les marges de manœuvre en matière de logement des personnes défavorisées sont réduites. Dans les 13 départements où le nombre de recours s'élève à plus de 50 par mois en moyenne sur la période allant de janvier 2008 à juin $2010^{16}$, les logements disponibles et accessibles en termes de loyers sont majoritairement situés dans les ZUS et les territoires visés par les CUCS. Sur le terrain, les objectifs en matière de droit au logement et de mixité sociale s'avèrent donc particulièrement difficiles à concilier. Les modalités de gestion de ces contradictions par les acteurs locaux contribuent paradoxalement à la concentration de l'offre de logement aux bénéficiaires du DALO dans les zones les plus pauvres, qui sont pourtant parfois ciblées par la politique de la ville.

\section{Un exemple de concentration de l'offre dans les « quartiers sensibles » : la Communauté Urbaine de Strasbourg}

Le territoire de la Communauté Urbaine de Strasbourg (CUS), qui accueille plus de $88 \%$ des recours devant les commissions de médiation DALO du département du Bas-Rhin ${ }^{17}$, a fait l'objet d'une analyse cartographique et statistique de la répartition socio-spatiale de l'offre d'habitat aux bénéficiaires du DALO entre 2009 et 2010. La première carte montre qu'à l'inverse des places d'hébergement (carrés noirs), plutôt situés en centre-ville, de nombreuses propositions de logement (carrés blancs) sont situées dans des ZUS en périphérie de l'agglomération, délimitées par une ligne gris foncé. La part des offres de logement dans ces " quartiers sensibles " s'élève à 35,9\% de l'ensemble de l'offre de logement aux ménages DALO, ce qui dépasse la limite des $30 \%$ fixée par le ministère. De surcroît, cette proportion représente plus du double de celle de la population de l'agglomération résidant en ZUS, qui ne s'élève qu'à $17 \%$ fin 2006. La concentration de l'offre aux ménages DALO sur ces territoires particulièrement denses - les ZUS s'étalent sur seulement $3 \%$ de la superficie du territoire de la CUS est d'autant plus importante que leur population a diminué de $3,5 \%$ entre 1999 et $2006^{18}$.

14. Entretien avec le Président de la Commission de médiation départementale DALO de Paris, septembre 2009.

15. Circulaire du ministère de l'Écologie, du Développement durable, des Transports et du Logement (MEEDAT) du 30 septembre 2008.

16. Durant l'année 2010, il s'agissait des Bouches-du-Rhône, Loire-Atlantique, Nord, Var, Rhône, HauteGaronne, Alpes-Maritimes, Hérault, Isère, Gironde, Oise, Bas-Rhin et Seine-Maritime. Ces départements renvoient souvent à la présence d'une agglomération de plus de 450000 habitants. Voir le $4^{e}$ Rapport du Comité de Suivi de la Mise en CEuvre du DALO, 2011.

17. Bilan 2008 de la commission de médiation DALO du Bas-Rhin, CETE de l'Est, 2009.

18. Rapport annuel de l'observatoire des ZUS, Agence de Développement et d'Urbanisme de l'agglomération strasbourgeoise, octobre 2011. 


\section{Localisation de l'offre d'habitat aux ménages prioritaires DALO sur le territoire de la CUS (2009-2010)}

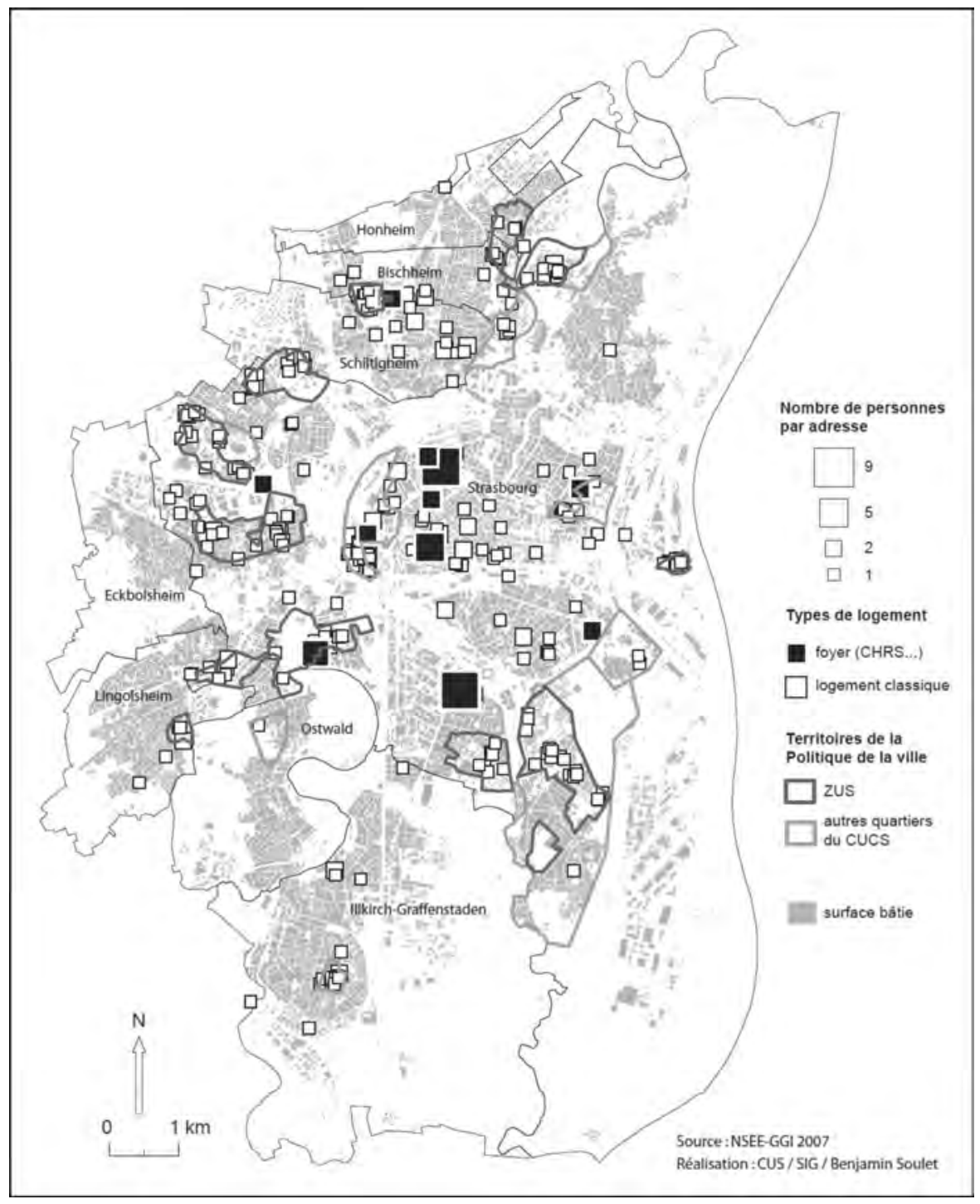

La localisation de l'offre de logement montre par ailleurs que de nombreuses propositions se situent dans des quartiers ciblés par le CUCS, mais qui ne sont pas classés ZUS (25,9\%). La mise en œuvre du DALO contrarie fortement les programmes de "rénovation urbaine " et les objectifs de peuplement de ces quartiers, mais ils apparaissent moins " prioritaires " que les ZUS et ne sont pas pris en compte dans la circulaire mentionnant l'objectif de réduction des risques de concentration des ménages DALO. Néanmoins, certains quartiers du CUCS situés hors ZUS ont un taux de chômage ou de logements sociaux supérieurs, et un revenu médian inférieur à ceux de certaines ZUS. À titre d'exemple, les ménages de 
la ZUS Koenigshoeffen-Ouest sont à $45,8 \%$ locataires de logements sociaux, ils ont un revenu moyen de 12630 euros par an et $22,3 \%$ d'entre eux sont concernés par une allocation chômage en 2009, tandis que ceux du quartier Liebermann, visé par le CUCS mais situé hors ZUS, sont à 96,9\% locataires de logements sociaux, disposent d'un revenu moyen de 11940 euros par an, et $26,5 \%$ d'entre eux sont concernés par une allocation chômage ${ }^{19}$. Par ailleurs, un nombre important de logements sont proposés dans des quartiers dont la proportion de logements sociaux est relativement élevée, mais qui ne sont pas ciblés par la politique de la ville. Les acteurs locaux des politiques de l'habitat les considèrent pourtant "en déclin ", de même que certains de leurs habitants. C'est le cas du quartier Esplanade, en lisière du centre-ville et du campus universitaire. Une " opération anti-terroriste " menée en octobre 2012 représentation alarmiste y attire en outre les caméras de télévision et nourrit une représentation stigmatisante, qui ressort notamment des interviews des riverains par une journaliste de Libération:

"Une dame blonde les rejoint d'un pas décidé, elle est très remontée contre le bailleur social qui possède les immeubles: "CUS habitat met n'importe qui ici, c'est dangereux". Elle réclame que des enquêtes soient faites avant d'attribuer les logements. "Quand la ville casse des barres d'immeubles au Neuhof ou à Hautepierre, ils relogent les gens ici. C'est un quartier que les politiques ont volontairement laissé se dégrader". Cet été sa voisine du dessus a "jeté son frigo par le balcon" et ce matin elle découvre que son "voisin était un terroriste", ç'en est trop, elle voudrait partir. Mais tout ce qu'on lui propose ce sont les nouveaux logements, construits justement au Neuhof ou Hautepierre ${ }^{20}$. "

Par ailleurs, la localisation des offres de logement et des places d'hébergement sur une carte du territoire de la CUS, découpé en îlots de population caractérisés par le revenu médian de leurs habitants, permet une analyse plus fine de leur répartition socio-spatiale. On constate ainsi que $81 \%$ des propositions de logement se situent dans des zones de l'agglomération où le revenu fiscal par habitant est inférieur à la médiane de la population. La quasi-totalité des offres localisées dans les îlots de population au revenu fiscal médian par unité de consommation de plus de $30 \%$ inférieur à celui de la communauté urbaine sont en ZUS ou dans d'autres quartiers ciblés par le CUCS. Néanmoins, $16 \%$ des logements proposés se situent dans des îlots de population dont le revenu fiscal médian par unité de consommation est inférieur de plus de $20 \%$ au revenu strasbourgeois, sans que ces zones ne soient pour autant visées par le CUCS. Cette carte montre ainsi que le peu d'offres de logement qui ne se situent pas dans des quartiers ciblés par la politique de la ville s'inscrivent tout de même dans les fractions les plus pauvres de l'agglomération. À l'inverse, les quartiers favorisés, où le revenu fiscal médian par unité de consommation dépasse de plus de $30 \%$ celui de la CUS, incluent pour ainsi dire uniquement des offres de place d'hébergement.

19. Voir les pages suivantes sur le site du système d'information géographique du Secrétariat général du Comité Interministériel des Villes [http://sig.ville.gouv.fr/Territoire/4201130] et [http://sig.ville.gouv.fr/Territoire/ 4267006], consultées le 21/08/2012.

20. Rousseau, 2012, consulté en ligne [http://www.liberation.fr/societe/2012/10/07/a-strasbourg-les-voisins-dejeremy-louis-sidney-cherchent-a-comprendre_851497 le 26 octobre 2012]. 


\section{Localisation de l'offre d'habitat aux ménages prioritaires DALO sur le territoire de la CUS (2009-2010)}

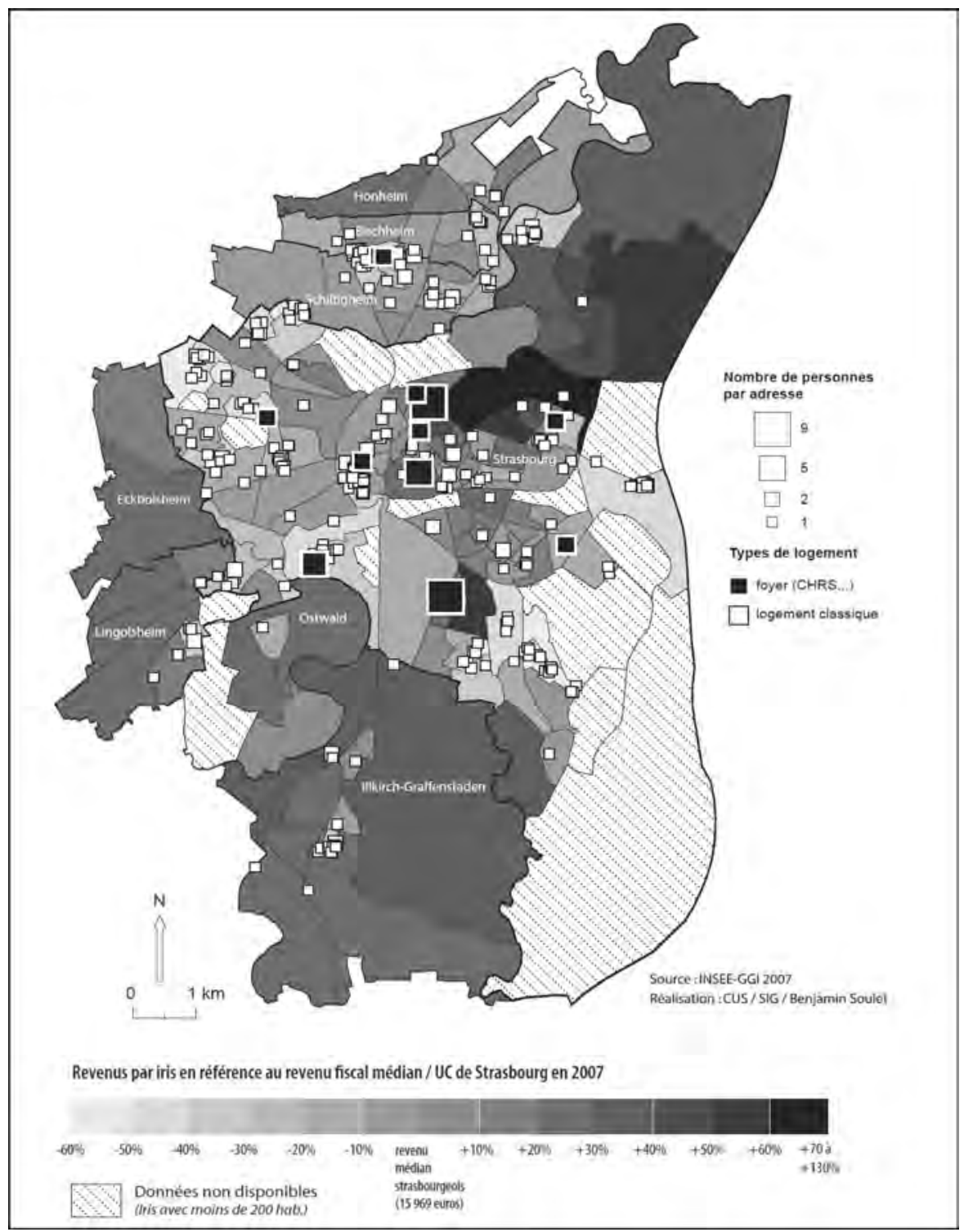


La concentration des personnes défavorisées dans les zones les plus pauvres de la CUS a partie liée au fait que les offices publics HLM (OPHLM) ${ }^{21}$, spécialisés depuis longtemps dans l'accueil des ménages dits « du bas du panier"22 " (Sala Pala, 2006 ; Gérard, 2011), prennent en charge la grande majorité des propositions de logement au titre du DALO (73 \%). Relèvent en effet de leur parc, souvent situé en ZUS, la grande majorité des réservations au titre du contingent préfectora ${ }^{23}$. Déjà traditionnellement affectées aux populations défavorisées, ces réservations sont désormais en grande partie affectées à la mise en œuvre du DALO. C'est notamment le cas de CUS Habitat, le principal OPHLM alsacien, que le préfet désigne le plus souvent pour accueillir les ménages DALO (34,9\% des propositions). Les acteurs de l'habitat proposent ainsi de nombreux logements aux bénéficiaires du DALO dans des quartiers de grands ensembles particulièrement paupérisés, comme la Cité Nucléaire de Cronenbourg, Neuhof-Cités ou Port du Rhin, où le revenu fiscal médian par unité de consommation est plus de $60 \%$ inférieur à celui de l'ensemble de la communauté urbaine. Les OPHLM accueillent en outre la majeure partie des $17 \%$ de ménages prioritaires dont les bailleurs sociaux privés ou semi-publics ${ }^{24}$ refusent les candidatures en commission d'attribution, tandis que l'inverse est rarissime. L'étiquetage DALO accentue en outre certaines craintes relatives à des populations "à risques ". Des courriers adressés aux services des administrations déconcentrées en charge du relogement, ou de simples notes sur un post-it dans un dossier témoignent ainsi des résistances d'un bailleur à accueillir des femmes célibataires avec des enfants adolescents comme premiers occupants d'un immeuble d'habitat social en fin de construction, de peur de voir celui-ci se dégrader trop rapidement ${ }^{25}$. Les bailleurs n'ont pas renoncé à défendre leurs objectifs de mixité sociale au sein des franges les plus attractives de leur parc, malgré la menace accrue d'une saisine de la Mission Interministérielle d'Inspection du Logement Social ${ }^{26}$ ou de voir le préfet user de son droit de substitution. Mais les bailleurs les plus réticents face à certains ménages prioritaires proposés par le préfet peuvent toujours arguer de leur insuffisance de ressources économiques pour refuser un logement. II en va de même pour les appartements qui relèvent des réservations des financeurs du $1 \%$ Logement ${ }^{27}$. En mars 2009, la Loi de mobilisation pour le logement et la lutte contre l'exclusion dite " MOLLE » portée par la ministre Christine Boutin, impose d' attribuer 25 \% de ces réservations à des salariés ou de chômeurs "prioritaires " au titre

21. Les offices publics HLM sont des établissements publics rattachés aux collectivités locales, gérés par un conseil d'administration composé de représentants des collectivités locales, de l'administration, des partenaires financiers et sociaux et des locataires. Ils construisent, gèrent et améliorent les logements destinés à la location et à l'accession à la propriété et interviennent en matière d'aménagement et d'urbanisme pour les collectivités publiques. Voir l'ordonnance $n^{\circ}$ 2007-137 du $1^{\text {er }}$ février 2007 relative aux offices publics de l'habitat.

22. Pour reprendre l'expression d'un salarié d'office public HLM rencontré en mai 2010.

23. Dans chaque département, les préfets disposent d'un droit de réservation, le " contingent préfectoral ", allant jusqu'à $30 \%$ des logements locatifs sociaux de chaque organisme bailleur.

24. On parle de sociétés anonymes d'habitations à loyer modéré lorsque les capitaux sont détenus par des personnes privées et de société d'économie mixte lorsqu'il existe une participation publique au capital, qui peut atteindre jusqu'à $85 \%$.

25. Matériaux consultés dans le cadre de l'observation ethnographique du travail administratif.

26. Administration nationale de contrôle de la gestion des organismes HLM, celle-ci est notamment en charge de la lutte contre la discrimination dans l'accès au logement social.

27. Le $1 \%$ Logement est la dénomination usuelle de la participation des Employeurs à l'Effort de Construction, instituée en 1953 pour les entreprises privées. À l'origine, celles-ci devaient consacrer $1 \%$ de leur masse salariale au financement de la résidence principale des salariés. Après plusieurs baisses successives, ce taux est fixé depuis 1992 à 0,45\%. 
du DALO ${ }^{28}$. Moins souvent situés dans des "quartiers sensibles", les appartements concernés requièrent toutefois des loyers plus élevés que ceux qui relèvent des réservations du contingent préfectoral, les rendant peu accessibles à ces ménages. Les réticences à l'égard des ménages cumulant le plus de difficultés sociales et économiques contribuent donc à la spécialisation d'une partie du parc social dans l'accueil des plus pauvres et la ségrégation spatiale. Cette évolution est d'ailleurs confortée par la constance du flux de nouveaux ménages prioritaires. Dans les zones où le marché locatif est le plus tendu, certains bailleurs ont bien compris qu'ils se trouvent toujours d'autres ménages DALO mieux lotis pour remplacer le premier candidat désigné par le préfet, et remplir les logements vacants de leur parc. Certains ménages apparaissent donc de fait plus "prioritaires » que d'autres, eu égard à leur composition familiale et à leur situation financière. Ils peuvent ainsi bénéficier de logements plus attractifs, souvent situés en dehors des "quartiers sensibles ", en centreville ou dans des quartiers périphériques dont le revenu moyen se rapproche ou dépasse légèrement la médiane de l'agglomération.

\section{La structuration de l'offre d'habitat proposée aux ménages DALO}

L'analyse du fichier de logement ou d'hébergement des ménages DALO sur le territoire de la CUS révèle la diversité des caractéristiques des ménages concernés et de leur habitat d'origine ${ }^{29}$, ainsi que des solutions de logement ou d'hébergement proposées. Or, on peut faire l'hypothèse, que la nature des solutions offertes est fortement liée à certaines caractéristiques des ménages et de leur situation reconnue comme " prioritaire " et " urgente ". L'observation ethnographique à la mission DALO de la DDCS du Bas-Rhin révèle que les éléments relatifs à la taille et la composition du foyer s'avèrent à la fois primordiaux et déterminants dans le travail de médiation des agents de l'État auprès des responsables de foyers ou des bailleurs sociaux qui disposent d'un logement à attribuer, et cela même avant que ne rentrent en compte les questions de revenus disponibles. Ces agents rencontrent notamment des difficultés à trouver des logements vacants qui « matchent » avec la situation des personnes seules, ou encore à reloger les " grandes familles » ailleurs que dans des quartiers de grands ensembles situés en ZUS, souvent les seuls à voir se libérer des appartements de type F5. II importe cependant de durcir ces hypothèses sur les logiques de la répartition socio-spatiale de l'offre, en distinguant les ressemblances et les oppositions entre les solutions de logement ou d'hébergement proposées, rapportées à l'habitat d'origine et la composition familiale des ménages concernés, au moyen d'une ACM. Concernant le choix des variables, les ménages bénéficiaires d'une proposition de relogement ou d'hébergement sur le territoire de la CUS, sont d'abord caractérisés en fonction des indicateurs disponibles sur le statut familial de leurs membres. Ensuite, le type d'habitat d'origine des ménages, comme celui de la destination proposée, est différencié selon son appartenance au parc social ou privé, ainsi que selon la catégorie du territoire où il se situe, qu'il s'agisse d'un quartier relevant du CUCS ou d'une zone non ciblée par la politique de la ville.

28. Loi n $2009-323$ du 25 mars 2009 de mobilisation pour le logement et la lutte contre l'exclusion.

29. Une semblable diversité des ménages dont la situation est désignée "prioritaire et urgente " émerge de l'analyse, étayée par des données plus détaillées, des dossiers d'instruction des recours devant les autres commissions de médiation DALO étudiées. 


\section{- Variables actives et modalités de l'ACM ${ }^{30}$}

$(\mathrm{N}=384)$

Composition familiale des ménages :

- Personne seule : $33,5 \%$

- Famille monoparentale : $26,5 \%$

- Couple sans ou avec 1, 2 ou 3 enfant(s) : $23,2 \%$

- Grand ménage de 6 individus et plus : 16,8\%

Type d'habitat d'origine :

- Structure d'hébergement (CHRS, Foyer ADOMA ou Jeunes Travailleurs, Hôtel social) : $24,5 \%$

- Sans-abri ou hébergés chez un tiers : $6,4 \%$

- Logement sur-occupé ou insalubre : $69,1 \%$

Type d'habitat proposé :

- Structure d'hébergement (CHRS, Foyer ADOMA ou Jeunes Travailleurs, Hôtel social) : $10,7 \%$

- Logement locatif social : $89,3 \%$

Localisation du logement d'origine :

- Logement dans un quartier du Contrat Urbain de Cohésion Sociale (CUCS) : 57,8 \%

- Logement dans un quartier hors Contrat Urbain de Cohésion Sociale (CUCS) : 42,2 \%

Localisation du logement proposé :

- Logement dans un quartier du Contrat Urbain de Cohésion Sociale (CUCS) : 61,8\%

- Logement dans un quartier hors Contrat Urbain de Cohésion Sociale (CUCS) : 37,2 \%

L'ACM met en évidence la polarisation des propositions d'offre de logement ou d'hébergement aux « ménages prioritaires ", en fonction de leur composition familiale et de leur habitat d'origine. Les deux premiers axes de l'ACM expliquent une très forte proportion de la variance de l'échantillon (68,3\%). Le premier axe oppose les familles monoparentales et les grands ménages à destination des ZUS ou des autres quartiers du CUCS, qui sont originaires de ces derniers, aux couples avec ou sans enfants à destination des quartiers hors CUCS, qui en sont originaires et hébergés chez un tiers. Le deuxième axe oppose quant à lui les personnes seules, issues d'une structure d'hébergement, aux couples et aux familles avec des enfants, qui se voient proposer un véritable logement. Si les personnes seules sont minoritaires dans l'échantillon (34,5\%), elles se distinguent nettement des autres ménages, dans la mesure où l'attribution d'un logement autonome et durable leur semble relativement fermée par rapport aux familles. À l'inverse, ces dernières se voient proposer un logement social dans $89 \%$ des cas. Ceci étant, les logements proposés aux ménages de grande taille sont très majoritairement situés en ZUS et dans les autres quartiers du CUCS (65,6 \%). II en va de même pour les familles monoparentales (66\%), le requérant étant dans la quasi-totalité des cas une mère célibataire. À l'inverse, les couples avec un ou deux enfants sont beaucoup moins souvent orientés vers les quartiers du CUCS (47,6 \% contre 61,8\% de l'ensemble de l'échantillon).

30. L'ACM porte sur un échantillon de ménages dont la situation a été considérée "prioritaire et urgente " par la commission de médiation du Bas-Rhin, et qui font l'objet d'une proposition de logement ou d'hébergement sur le territoire de la CUS. 


\section{Habitat, territoire et composition familiale}

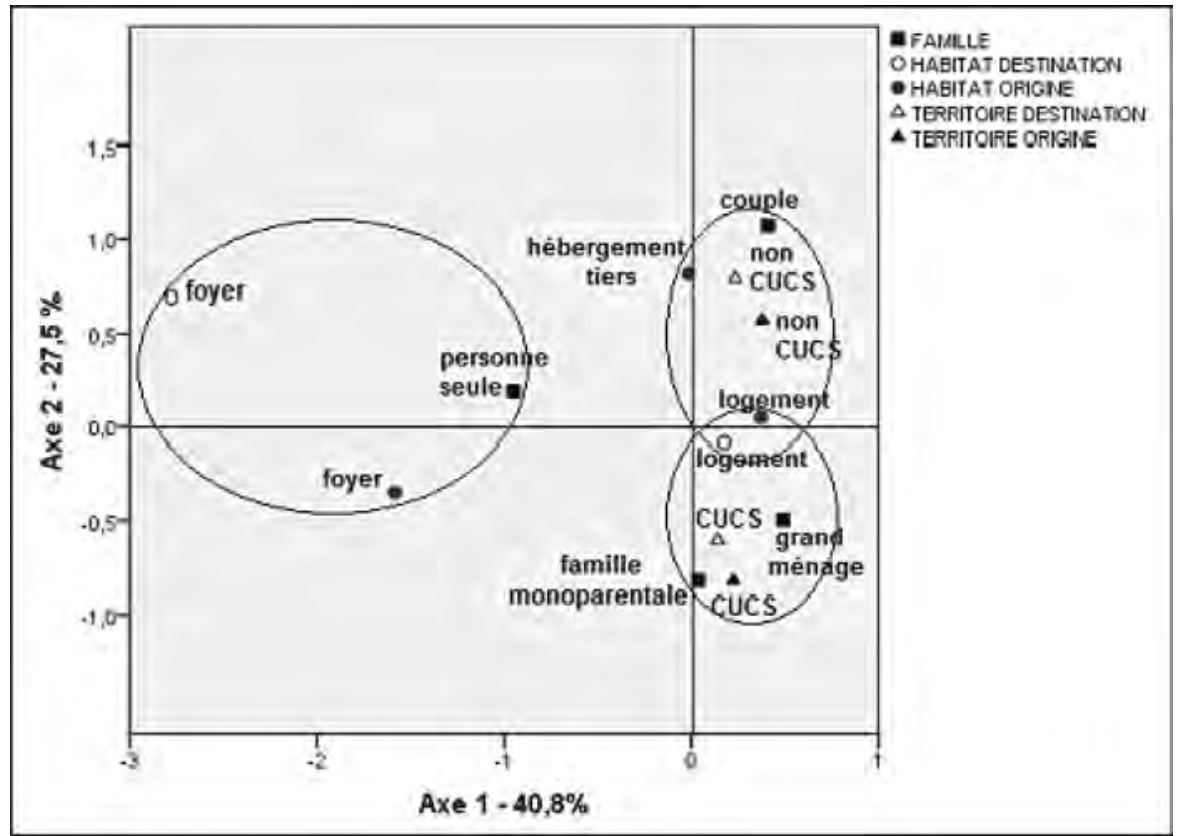

Source: Auteur.

Non disponibles pour une grande partie des ménages figurant sur l'extrait de fichier administratif exploité, les propriétés sociales autres que la composition familiale, n'ont pas été prises en compte dans l'ACM. L'analyse des données des dossiers d'instruction des recours devant la commission de médiation DALO du Bas-Rhin offre cependant un éclairage complémentaire sur les logiques de différenciation de l'offre. On sait notamment que le niveau de vie de $77 \%$ des requérants se situe sous le seuil de pauvreté, mais que des revenus économiques majoritairement bas masquent de fortes disparités en terme de composition familiale, d'âge et d'activité professionnelle. Les personnes seules comptent par exemple un taux d'inactifs particulièrement fort (62\%). Nombre d'entre eux sont retraités, perçoivent un RSA socle ou une allocation handicap, lorsqu'ils disposent de revenus déclarés, et ne se voient proposer qu'une offre d'hébergement, leur recours ayant été requalifié par anticipation du refus des bailleurs sociaux de les accueillir. À l'inverse, les couples avec un maximum de 3 enfants disposent généralement de revenus supérieurs au reste des requérants. Leurs ménages comportent généralement un ou deux actifs, qui occupent des emplois souvent précaires et peu qualifiés, mais dont les revenus, ajoutés aux prestations familiales, les rendent comparativement plus solvables que les autres bénéficiaires du DALO. Ils sont dès lors plus susceptibles d'être orientés vers les franges les moins paupérisées du parc social. Ils proviennent d'ailleurs le plus souvent de territoires non ciblés par les politiques de la ville, dont le revenu fiscal médian par unité de consommation se rapproche, voire dépasse celui de l'agglomération. Les couples avec 1 ou 2 enfants s'opposent ainsi aux familles nombreuses, fréquemment issues des centres d'hébergement ou de logements situés en ZUS ou dans les autres quartiers du CUCS. Les agents de l'État leur proposent en effet des appartements peu onéreux, en capacité de les accueillir. Situés pour la plupart en ZUS, ils sont en effet caractérisés par un fort taux de 
turn-over. Si les caractéristiques des ménages et de leur situation en matière d'habitat conditionnent l'offre d'action publique, il s'agit désormais de montrer comment elles déterminent aussi ses réceptions socialement contrastées.

\section{Les appropriations socialement différenciées de trajectoires sous contraintes}

Les propositions de logement ou d'hébergement au titre du DALO ne sont généralement pas négociables. Or, leur important taux de refus ne manque pas de surprendre et d'interroger les acteurs du logement des personnes défavorisées, qui tendent à en rejeter la responsabilité sur les ménages concernés. Afin de dépasser les jugements moraux ou les explications individualisantes de certains acteurs institutionnels, on peut distinguer des profils spécifiques de bénéficiaires du DALO, dont les caractéristiques les plus saillantes sont liées à des appropriations socialement différenciées de l'offre d'action publique.

\section{L'étonnement institutionnel face au refus de l'offre}

L'offre de logement aux ménages " prioritaires " constitue la dernière étape d'une procédure administrative parsemée de difficultés. En outre, la proposition fait parfois suite à de nombreuses années d'attentes d'un logement social, certaines familles étant hébergées chez un tiers ou dans un centre d'hébergement depuis plus de 18 mois. Elle répond aussi souvent à une situation qualifiée de " prioritaire et urgente " par les acteurs locaux du logement des personnes défavorisées, en raison de la suroccupation ou de l'insalubrité de leur habitation, lorsque le ménage ne doit pas faire face à un jugement d'expulsion accompagné d'un ordre d'intervention de la force publique. Autant de motivations du recours qui alimentent un constat d'étonnement partagé face au refus de certaines propositions, qui s'exprime jusqu'au plus haut niveau. Interrogée sur les 25,3\% de refus des offres de logement enregistrés après 9 mois de mise en œuvre du DALO, Christine Boutin, déclare ainsi qu'au ministère, " on est surpris par ce phénomène de refus. Certaines raisons sont recevables, d'autres plus surprenantes, de l'ordre des convenances personnelles ${ }^{31}$ ». Au niveau départemental, le Centre d'Études Techniques de l'Équipement de l'Est conclut son bilan sur le fonctionnement de la commission DALO sur la question :

"Le nombre très important de refus suite aux propositions de relogement étonne et interpelle les acteurs de la procédure. Une analyse fine de ces refus est attendue par ceux-ci afin d'en déterminer le problème (requérant trop exigent, situation non urgente, proposition inadaptée, mauvaise foi du requérant) ${ }^{32}$. ”

Le refus massif des places en centres d'hébergement suscite des interrogations sur la nature de l'offre chez certains agents de l'État local, qui admettent leur éloignement du quotidien des «sans-abris » en commission de médiation :

[ "On devrait aller visiter les structures d'hébergements, c'est vrai que je ne connais pas assez... ${ }^{33}$ " $]$

31. Interview de Christine Boutin, Secrétaire d'État au Logement, radio RMC, octobre 2008.

32. Bilan 2008 de la commission de médiation DALO du Bas-Rhin, CETE de l'Est, 2009.

33. Extrait d'une réunion de commission de médiation, mai 2009. 
En revanche, les acteurs de l'habitat placent plus volontiers la responsabilité des refus du côté des demandeurs, lorsqu'il s'agit de propositions de logement. Les refus ne manquent d'ailleurs pas de susciter l'irritation des acteurs de terrain du logement des personnes défavorisées. Un responsable du service habitat social de la CUS ironise par exemple sur le fait que "certains refusent parce que l'armoire normande de famille ne passe pas par la porte d'entrée ${ }^{34} »$. Un agent de la DDCS du Bas-Rhin exprime quant à lui clairement sa frustration :

"Quand vous vous démenez pour trouver un logement pas trop cher dans la ville qu'elle souhaite à une personne en difficulté et qu'elle vous raconte 'Ah bah, là ça va pas! Mon canapé va pas passer !'. Alors qu'ils vivent dans un taudis ! Alors qu'on s'est décarcassé pour le petit bonhomme ${ }^{35}$ ! »

La proportion importante de refus alimente de surcroitt la suspicion des bailleurs à l'égard des ménages DALO. Certains dénoncent ouvertement une « filière parallèle ${ }^{36}$ » et « ont le sentiment que les requérants pensent bénéficier d'un passe-droit ${ }^{37}$ ". Ils accusent " la méconnaissance de certains requérants de ce à quoi leur ouvre droit le DALO ${ }^{38}$ ", qu'il s'agisse des caractéristiques du logement proposé ou du montant du loyer :

"Certains sont vraiment naifs ou de mauvaise foi. lls nous disent : Ah bon, il y a donc un loyer ! Mais je croyais que j'avais le droit au logement ! Ah bah, dans ces conditions j'en veux pas en fait ${ }^{39}$. "

Ces anecdotes et ces récriminations illustrent les représentations des acteurs de terrain des «convenances personnelles" dénoncées par la ministre. Elles relèvent cependant du cas par cas et de représentations psychologisantes, qui n'épuisent guère l'explication des attitudes des ménages ayant recours au DALO face à l'offre d'action publique.

\section{Donner une seconde chance : les conditions sociales de la grâce}

Les conséquences des refus peuvent se révéler particulièrement graves pour les ménages concernés, à la mesure de la sévérité des jugements moraux que certains professionnels de l'habitat portent sur eux. La loi impose en effet la proposition d'un logement adapté, mais les citoyens ne disposent pas, en théorie, du choix de le refuser, sous peine de perdre leur statut de "prioritaire ». Néanmoins, les membres de la commission peuvent accorder à certains ménages une seconde chance, suite à un refus de logement au motif, pourtant juridiquement illégitime, de la réputation du quartier. L'accord d'une telle grâce joue alors sur la marge de manœuvre accordée par la loi. Cette marge de manœuvre renvoie cependant à une pragmatique du jugement, qui relève de considérations moins juridiques qu'éthiques, ou économiques. Suite à la discussion d'un cas de recours gracieux en réunion, le président de la commission de médiation du Bas-Rhin décide par exemple de « donner

34. Entretien avec un responsable d'office public HLM, octobre 2009.

35. Entretien avec un agent de la DDCS du Bas-Rhin, septembre 2009.

36. Entretien avec un membre de commission de médiation, février 2009.

37. Bilan 2008 de la commission de médiation DALO du Bas-Rhin, CETE de l'Est, 2009.

38. Idem.

39. Pierre Carli, Président de l'AORIF, $4^{\circledR}$ bilan parlementaire sur le DALO, Table ronde "Après le passage en commission ", 30 novembre 2011. 
une seconde chance à une famille qui veut s'en sortir » et le justifie ouvertement par des arguments relatifs à la "moralité " du requérant concerné : "Bon vous voyez (le président se tourne vers moi) c'est un acte administratif mais on peut parfois laisser parler notre cœur, c'est une question de morale $!^{40}$ ». Interrogé sur ce cas, un agent de l'État local justifie la décision de la commission de ne pas respecter la Loi au pied de la lettre, en passant outre ce premier refus de relogement : "Là c'est du pragmatisme! C'est pas du droit, parce que le Neuhof c'est particulier... ${ }^{41}$ ». Dans les Yvelines, le cas d'un ménage ayant justifié un refus de relogement pour un motif similaire, fait l'objet d'un débat en commission, afin de savoir s'il doit être de nouveau désigné à un bailleur. Ce cas est tranché favorablement, à raison d'un argument plus prosaïque que dans le cas précédent : "Ça m'embête ce refus, mais y a les ressources, donc un bailleur finira bien par les prendre... ${ }^{42}$ ". Depuis mars 2011, un décret clarifie toutefois les modalités de perte du statut de "prioritaire " et celles de sa justification au requérant, en cas de refus d'une proposition motivé par un critère extrajuridique. II s'agit d'aller dans le sens d'une plus grande rigueur à l'encontre des bénéficiaires du DALO qui manifestent leur insatisfaction. La fréquence des grâces s'avère en effet proportionnelle au taux de refus des propositions de logement, qui augmente au fur et à mesure de la mise en œuvre du dispositif, même s'il enregistre des variations départementales : il est légèrement supérieur à Strasbourg ou dans d'autres grandes villes de Province qu'à Paris, où les ménages prioritaires sont plus souvent "sans logis " que "mal logés ". La régionalisation du dispositif, qui permet de loger un requérant dans un autre département que celui où il a fait ses demandes de logement social, fait cependant craindre un regain de refus en Île-de-France.

De manière générale, le taux des refus des propositions de logement et d'hébergement des bénéficiaires du DALO constitue une préoccupation croissante des acteurs institutionnels. Ceux qui prennent en charge l'accompagnement social et juridique des familles s'efforcent de maintenir les ménages dont la situation est classée « prioritaire et urgente » dans le " droit chemin » de la procédure de relogement. Selon une responsable d'association d'insertion qui siège en commission de médiation : "(les) partenaires habituels ne vont plus être en mesure de nous procurer des logements, la plupart étant réquisitionnés dans le cadre de l'application de la loi DALO ${ }^{43}$ ". En précipitant le demandeur vers la sortie du dispositif mis en place dans le cadre de la loi DALO, le refus tend aussi à les exclure des dispositifs de droit commun. Versé à un dossier partagé par les différentes administrations concernées, il les suit et les stigmatise dans toutes leurs démarches de demande de logement social. Pour les acteurs associatifs, il s'agit donc d'avertir les requérants des conséquences du refus, pour mieux en prévenir les risques dès le début de la procédure. Ils travaillent ainsi à la production du consentement (Dubois, 2010 [1999]), tâchant de convaincre les bénéficiaires du DALO d'accepter des trajectoires résidentielles qui restent manifestement trop contraignantes pour certains.

40. Extrait d'une délibération de réunion de la commission de médiation du Bas-Rhin, Strasbourg, mai 2009.

41. Entretien avec un agent de la DDCS du Bas-Rhin, mai 2009.

42. Extrait d'une réunion de la commission de médiation des Yvelines, Versailles, novembre 2010.

43. Interview d'une responsable FNARS, 20 minutes, 10 octobre 2008. 


\section{Des profils spécifiques : comprendre et expliquer l'acceptation ou le refus de l'offre}

Les appropriations différenciées de l'offre d'action publique peuvent être examinées à l'aune des caractéristiques sociodémographiques et de la situation en matière d'habitat des ménages DALO. II s'agit en cela de mieux comprendre et expliquer les logiques de l'acceptation ou du refus des propositions de logement ou d'hébergement, en interrogeant leurs fondements sociaux. Les données offertes par le fichier de relogement des ménages sur le territoire de la CUS peuvent là encore être mobilisées. On ajoute cependant aux variables précédemment utilisées dans l'ACM - le type d'habitat d'origine et de destination proposée ainsi que la composition familiale - l'acceptation ou le refus des offres de logement et d'hébergement des ménages. Une typologie de ménages caractérisés par des configurations de modalités de ces variables, peut alors être établie à l'aide d'une classification ascendante hiérarchique.

II s'agit ensuite, pour chacune des classes distinguées de dégager des profils moyens, dont les traits les plus saillants font ressortir des liens entre la composition familiale des ménages, leur habitat d'origine, la destination proposée et l'acceptation ou le refus de cette dernière. On peut aussi mesurer les écarts de chacune des variables caractéristiques de ces profils de classe à leur moyenne dans l'ensemble de l'échantillon, afin de dégager des tendances à la fois compréhensives et explicatives des offres d'hébergement ou de logement, ainsi que de leurs réceptions différenciées. De même que le codage des données et le choix des variables se fondent sur une enquête ethnographique auprès d'acteurs institutionnels et de requérants DALO, l'usage de matériaux qualitatifs - des entretiens avec des ménages prioritaires, des comptes rendus de visites d'appartements ou des courriers de motivation de refus d'attribution de logement - contribue à enrichir les résultats de l'analyse quantitative. En effet, l'usage de ces matériaux donne à la fois de la chair aux regroupements d'individus statistiques, et du sens aux relations entre les modalités caractéristiques des ménages. L'interprétation de la construction statistique en fonction du terrain observé implique ici de distinguer 4 classes de ménages DALO aux effectifs relativement homogènes. Ces classes renvoient à des caractéristiques familiales et des situations en matière d'habitat variées, ainsi qu'à des attitudes contrastées face à l'offre d'action publique. Certains ménages n'accèdent que rarement au logement, bien qu'ils soient "prioritaires", car ils sont réorientés vers l'hébergement ou refusent l'offre qui leur est proposée pour des motifs variés. D'autres l'acceptent plus souvent, mais cette acceptation est plus ou moins synonyme de résignation ou de satisfaction.

\section{Les « assignés à résidence »}

La première et la plus importante des classes distinguées rassemble 29,8\% des ménages de l'échantillon. Cette classe se compose d'une forte majorité de personnes seules $(56,1 \%$ contre $34,7 \%$ ) et se caractérise principalement par le fait que $81,6 \%$ des ménages qui en relèvent sont issus d'un centre d'hébergement, contre seulement 24,3\% de l'ensemble de l'échantillon. Ceux-ci ont cependant tendance à être "assignés à résidence ». Bien que leur situation soit considérée comme "prioritaire » et « urgente », ils 
sont souvent réorientés vers une solution d'hébergement $(35,1 \%$ contre 10,5\% de l'ensemble des ménages observés), que la grande majorité d'entre eux refusent (61,5\%). Les requérants de cette classe ont fait les démarches nécessaires pour faire valoir leur droit au logement, le plus souvent suite à une période de résidence de plus de 18 mois dans une structure d'accueil. Or, les acteurs institutionnels les destinent souvent à occuper une place en Foyers de Jeunes Travailleurs (FJT) ou aux résidences ADOMA ${ }^{44}$, pour ceux qui occupent un emploi, des Centres d'Hébergement ou de Réinsertion Sociale (CHRS) pour les plus précaires, ou des résidences adaptées, pour les handicapés ou les retraités. Déclaré prioritaire, un ouvrier à la retraite âgé de 70 ans, qui vit seul dans une chambre de bonne au septième étage d'un immeuble sans ascenseur et qui se trouve en situation d'expulsion, manifeste par exemple son désir de rester dans un «appartement à lui ", et il refuse absolument de se voir placé dans une résidence sociale pour vivre en collectivité ${ }^{45}$. En outre, ceux qui font l'objet d'une proposition de logement s'en satisfont plutôt moins que les autres (39,2 \% contre 36 \% de refus parmi l'ensemble des ménages de l'échantillon qui se voient proposer un logement). Parmi les appartements individuels proposés aux ménages de cette classe, 59,5\% d'entre eux sont situés dans les quartiers du CUCS. Or, ils ne comblent pas toutes les aspirations de mobilité résidentielle, souvent renforcées par une longue attente. C'est notamment le cas d'un ouvrier algérien de 48 ans, résident d'un foyer ADOMA depuis 9 ans, dont la procédure de regroupement familial avec sa femme et ses trois enfants est en cours. II repart en effet insatisfait de la visite d'un appartement dans un quartier du centre-ville en cours de gentrification, proche de la gare centrale, car il le trouve «mal située ${ }^{46}$.

\section{Les « résignés »}

Dans la seconde classe, qui rassemble $23 \%$ de l'échantillon, les ménages se résignent plus souvent à l'offre de logement qui leur est proposée, même lorsque ce dernier se situe dans un quartier ciblé par le CUCS $(75 \%$ contre $61,8 \%$ des logements proposés à l'ensemble des ménages). Le fait que les ménages de cette classe résident tous déjà dans les quartiers du CUCS (tandis que 74,9\% de l'ensemble des ménages de l'échantillon n'en sont pas originaires), où ils vivent le plus souvent dans un appartement insalubre ou « suroccupé ", explique en partie pourquoi ils refusent moins souvent l'offre de logement qui leur est proposée (26,1\% contre $36 \%$ ). Cette classe comporte aussi un taux légèrement supérieur de familles monoparentales $(30,7 \%$ contre $28,5 \%)$ et rassemble une plus forte proportion de grands ménages de six individus ou plus (29,5\% contre $19,1 \%)$, pour lesquels les appartements adaptés disponibles sont difficiles à trouver en dehors des "quartiers sensibles ». Comme les chefs de familles modestes issues des dernières vagues d'immigration, qui bénéficient d'offres de relogement dans le cadre des politiques de lutte contre l'insalubrité, ces ménages DALO tendent à considérer la possibilité de se voir attribuer un logement social comme une chance, plutôt qu'un véritable droit (Dietrich-Ragon, 2011).

44. ADOMA, anciennement Société nationale de construction de logements pour les travailleurs (SONACOTRA), est une société d'économie mixte qui construit et gère de nombreux foyers de travailleurs migrants.

45. Observation participante à la permanence d'accompagnement juridique d'une association, traitement du dossier, avec son neveu, venu le représenter, décembre 2010.

46. Entretien avec un agent de l'agent local en charge du logement et consultation d'une copie du compte rendu de la visite du logement. 
Souvent issues de pays d'Afrique subsaharienne ou de zones de conflit d'Europe de l'Est, dont les ressortissants représentent $57,6 \%$ des ménages de plus de trois enfants bénéficiant d'une proposition de logement, contre $40,1 \%$ de l'ensemble des requérants DALO du BasRhin, ces familles ont tendance à cumuler les difficultés économiques et sociales. Elles acceptent plus facilement des offres de logements situés dans des quartiers populaires à forte concentration d'immigrés, faisant l'objet de refus de la part de ménages plus aisés, et où, selon les dires du député du Bas-Rhin, ancien président de CUS-Habitat, qui s'exprime devant le Conseil des résidents étrangers strasbourgeois, "les gens qui s'installent sont ceux qui ne peuvent aller nulle part ailleurs ${ }^{47}$ ". C'est par exemple le cas d'une jeune mère de famille de nationalité ivoirienne, sans activité salariée :

« J'essaye partout de toute façon, je tente ma chance avec le DALO là, mais j'y crois pas beaucoup franchement ! (...) Et puis voilà de toute façon ça ne peut pas continuer comme ça, il faut trouver un appartement là vraiment ! Non j'en peux plus là... à l'hôtel on peut pas faire la cuisine, rien ${ }^{48}$. "

Lorsque ces familles sont hébergées dans un CHRS, un foyer de transition ou un hôtel à vocation sociale, la situation est souvent très mal vécue, et le refus de l'attribution d'un logement autonome leur semble difficilement envisageable, comme chez ce père de famille mauricien, ouvrier du bâtiment au chômage :

"C'est pas pour vous faire pitié Monsieur, mais franchement je voudrais que ma fille sache ce que c'est d'avoir sa propre salle de bain ${ }^{49}$."

Ces ménages sont aussi les plus fortement pris en charge par des travailleurs sociaux ou des acteurs du monde associatif, à qui ils délèguent l'exercice des savoir-faire nécessaires à leur démarche. Or, ceux-ci les encouragent en retour à accepter les offres de logement proposées.

\section{Les « satisfaits »}

La troisième et la plus petite des classes de l'échantillon, qui rassemble $21 \%$ des ménages observés, ne comporte elle aussi que des bénéficiaires d'une offre de logement. Mais à la différence des ménages de la précédente classe, ceux-ci sont originaires de quartiers hors CUCS. Ils louent généralement des appartements insalubres ou sur-occupés du secteur privé dans des zones résidentielles relativement favorisées. Or, ils ont tendance à parvenir à demeurer en dehors des « quartiers sensibles ", tout en accédant à un logement social, augmentant ainsi leur confort résidentiel. Pour les ménages de cette classe, l'accomplissement de la procédure DALO permet donc plus souvent une trajectoire résidentielle ascendante. En cela, ils apparaissent comme les ménages les mieux satisfaits de l'action publique menée en leur faveur, et leur taux de refus des propositions de logement se révèle

47. Retranscription d'une conversation avec une habitante, Table ronde sur l'égalité d'accès au logement pour tous, Conseil des résidents étrangers, Foyer des étudiants catholiques, 25 octobre 2012.

48. Observation participante à la permanence d'accompagnement juridique d'une association, traitement du dossier, décembre 2010.

49. Observation participante à la permanence d'accompagnement juridique d'une association, traitement du dossier, février 2011. 
inférieur à celui de l'ensemble de l'échantillon (33,2 \% contre $36 \%$ ). Cela peut être lié au fait que cette classe est très majoritairement composée de familles $(77,5 \%$ contre $65,2 \%)$, parmi lesquelles on relève une plus forte proportion de couples avec un ou deux enfants (28,9 \% contre 21,7\%). En effet, ces ménages comportent généralement un ou deux actifs, qui occupent des emplois souvent précaires et peu qualifiés, mais dont les revenus ajoutés aux prestations familiales les rendent comparativement plus solvables que les autres familles ou personnes isolées. Ce qui peut s'en ressentir du côté de l'offre de logements, qui se situent plus souvent en dehors des "quartiers sensibles ", et dans des franges moins paupérisées du parc social que ceux qui sont proposés aux ménages des autres classes. À propos d'un couple strasbourgeois et de leurs deux enfants - le mari est magasinier et la femme fait des ménages chez des particuliers - qu'elle a accompagnés dans leurs démarches pour quitter un appartement insalubre, dont le propriétaire refusait de réaliser les travaux nécessaires, une assistante sociale évoque ainsi « la reconnaissance et le bonheur retrouvé d'une famille qui a été relogée dans un appartement du centre-ville ». Elle ajoute par ailleurs que «quand on propose quelque chose en centre-ville ils (les familles) s'y tiennent $!^{50}$ ".

\section{Les « déçus »}

À l'inverse, les ménages de la quatrième et dernière classe, qui rassemble 26,2\% de l'échantillon, ont plutôt tendance à être " déçus » du relogement par l'intermédiaire de la procédure DALO. Comme les familles de la précédente classe, dont elles se rapprochent en termes de composition, présentant même un taux légèrement inférieur de familles monoparentales (26,3\% contre $28 \%$ ), elles sont exclusivement originaires de quartiers de la CUS qui ne sont pas ciblés par l'intervention de la politique de la ville. En revanche, les ménages de la quatrième classe sont tous orientées vers des ZUS ou d'autres quartiers du CUCS, et sont caractérisées par un taux de refus des offres de logement supérieur à celui de l'ensemble de l'échantillon (44,1\% contre $36 \%$ ). Les aspirations de mobilité résidentielle de ces familles se révèlent donc souvent déçues par les propositions qui leurs sont faites, quand bien même elles se trouvent sans logis, ou à payer le loyer d'un appartement insalubre et dangereux. En effet, ces ménages refusent d'autant plus massivement les logements proposés qu'ils résident dans des quartiers moins paupérisés et stigmatisés que ceux qui sont ciblés par la politique de la ville. Dans une lettre venant à l'appui d'un recours gracieux devant la commission de médiation du Bas-Rhin, suite à la perte du statut de «prioritaire " liée à un refus de logement en ZUS, une mère de famille raconte par exemple sa visite d'un appartement d'un grand ensemble du quartier de Neuhof-Cités en insistant sur « les boîtes aux lettres brûlées dans la cage d'escalier ». Elle conclut son courrier en affirmant qu'«il est impossible d'élever des enfants dans un quartier pareil $»^{51}$. D'autres motifs de refus liés à l'environnement du quartier peuvent être invoqués par les personnes seules, qui représentent $25 \%$ de cette classe. On trouve notamment des retraités qui font l'objet d'une proposition de relogement dans un autre quartier que celui où ils résident depuis parfois plusieurs dizaines d'années. Aussi mal logés soient-ils, ces prioritaires DALO souhaitent conserver leurs habitudes quotidiennes. L'absence

50. Entretien avec une assistante sociale d'un centre communal d'action sociale (CCAS), Strasbourg, septembre 2009.

51. Lettre de recours gracieux présentée et discutée en réunion de la commission DALO du Bas-Rhin, Strasbourg, mai 2009. 
de transports en commun adaptés, pour des actifs qui travaillent tôt le matin dans la sécurité, le bâtiment ou le ménage, qui représentent 34 \% des requérants prioritaires, est par ailleurs souvent invoquée en région parisienne. C'est ainsi le cas d'un chauffeur routier qui travaille à Paris-Bercy, à qui on a proposé un appartement de type T3 à Noisy-le-Sec en Seine-SaintDenis, d'une superficie adaptée pour sa femme enceinte et leurs deux enfants :

"L'appartement me convenait parfaitement. Mais le problème, c'est que je commence à travailler à $5 \mathrm{~h}$ du matin, je n'ai pas de voiture et la première navette est à $6 \mathrm{~h} 42^{52}$. ”

Ce dernier motif est cependant beaucoup moins souvent mis en avant dans une agglomération de taille moyenne comme Strasbourg, bien desservie par les transports en commun, y compris dans les zones les plus défavorisées.

\section{Conclusion}

La mise en œuvre du DALO ne saurait se résumer à un "coup d'épée dans l'eau ${ }^{53}$ ". Elle montre au contraire ce qu'une obligation de résultat fondée sur la mobilisation du droit des administrés recèle de conséquences parfois inattendues, dans un contexte de retrait de l'État-providence.

En se substituant à une obligation de moyens fondée sur des contrats dont le contenu n'est pas toujours respecté par les pouvoirs locaux, une obligation de résultat garantie par l'État favorise la reprise en main d'un domaine d'action publique fortement territorialisé. Un « droit au logement » restreint, mais au contenu juridique fort, chasse alors progressivement la norme de "mixité sociale ", au contenu juridique faible, comme principe structurant de la gestion du parc social. II le fait d'autant mieux que son application constitue un indicateur de la "demande sociale" et des performances mises en œuvre pour la combler. Depuis 2008, le taux de relogement des ménages DALO contribue à justifier le financement du programme « Développement et amélioration de l'offre de logement » des lois de finances. II détermine aussi en partie la répartition des " aides à la pierre » entre les départements, celles-ci s'étant littéralement écroulées depuis la fin des années 1980, elles sont divisées par cinq entre 1988 et 2010 et perdent encore 46,6 \% entre 2010 et $2012^{54}$. Or, cette comptabilité des besoins « urgents et prioritaires » de logement présuppose une généralisation du modèle de l'acteur rationnel, qui s'avère peu conforme à la réalité : de nombreux individus n'ont pas recours au dispositif ou se révèlent incapables de faire valoir leur droit, bien qu'éligibles au regard de la loi. Elle exclut aussi les ménages "déçus ", souvent parmi les moins défavorisés, dont les aspirations socialement situées en matière de logement en font les plus susceptibles de refuser certaines offres de logement situés dans des quartiers stigmatisés. Leur attitude participe alors indirectement à

52. Interviewé par un journaliste de la radio $R M C, 17$ octobre 2008.

53. "Logement : un coup d'épée DALO ? ", L'Humanité, mardi 2 décembre 2008 ; « La loi Dalo, un coup d’épée dans l'eau ? ", 20 minutes, 29 avril 2008.

54. Rapport thématique de la Cour des Comptes, Les aides à la Pierre, mai 2011 ; Projets de performances "Ville et logement » annexés aux projets de lois de finances 2011 et 2012. 
renforcer une conception résiduelle du logement social, qui serait réservé à ceux qui ne peuvent vraiment pas accéder au marché locatif privé.

Nouvelle forme de gestion de la pénurie, la mise en œuvre d'un droit opposable ne vient toutefois que confirmer l'appauvrissement des candidats à l'accès au parc social. Elle s'inscrit dans un mouvement plus large et plus ancien de paupérisation de ces occupants - la part des ménages appartenant au quart de la population aux plus faibles revenus ayant évolué de $12 \%$ en 1973, à 39,8 \% en $2006^{55}$. L'orientation majoritaire des ménages DALO vers les quartiers ciblés par la politique de la ville et les programmes de « rénovation urbaine » accentue les difficultés d'accès à d'autres territoires d'habitat de familles défavorisées, nombreuses ou monoparentales et souvent issues de l'immigration. Au-delà des difficultés à concilier droit au logement et " mixité sociale ", la concentration des familles les plus démunies dans les «quartiers sensibles » contribue à justifier et perpétuer leur catégorisation. En fin de compte, la gestion, ou plutôt la digestion des contradictions entre droit au logement et mixité sociale participe ainsi au renforcement de la spatialisation de la question sociale (Poupeau, Tissot, 2005).

\author{
Pierre Édouard Weill \\ Institut d'études politiques \\ Université de Strasbourg \\ pierreedouard.weill@gmail.com
}

\title{
Bibliographie
}

Bacque, M.-H., Fijalkow, Y., Flamand, A., VerMEERSCH, S. (2010), "Comment nous sommes devenus HLM. Les opérations de mixité sociale à Paris dans les années 2000 ", Espaces et Sociétés, 140-141, p. 93-111.

Belorgey, N. (2011), "Réduire le temps d'attente et de passage aux urgences". Une entreprise de "réforme" d'un service public et ses effets sociaux", Actes de la recherche en sciences sociales, 189, p. 16-33.

Bourdieu, P. (1986), "La force du droit. Pour une sociologie du champ juridique", Actes de la recherche en sciences sociales, 64, p. 3-19.

BouRGEOIs, C. (2012), "Quand expertise, normes et délibérations conditionnent l'accès aux droits sociaux: la cas des commissions Droit au logement ", dans Bureau, M.-C., Sainsaulieu, I. (dir.), Les Configurations pratiques de l'État social, Paris, Septentrion, p. 133-151.

BRuneteaux, P. (2007), « Les politiques de l'urgence à l'épreuve d'une ethnobiographie d'un SDF ", Revue française de science politique, 57 (1), p. 47-67.
Damon, J. (2002), La Question SDF. Critique d'une action publique, Paris, PUF, coll. "Le Lien social».

Dietrich-Ragon, P. (2011), Le Logement intolérable, Paris, PUF, coll. « Le lien social ».

DuBoIs, V. (2010) [1999], La Vie au guichet. Relation administrative et traitement de la misère, Paris, Economica, coll. "Études politiques ».

FouCAULT, M. (1977), « La redéfinition du judiciable », Justice, 115, p. 36-39.

GAXIE, D. (1997), Luttes d'institutions. Enjeux et contradictions de l'administration territoriale, Paris, L'Harmattan, coll. "Logiques juridiques ».

GÉRARD, B. (2011), "L'évolution de la mixité sociale, enjeu du renouvellement urbain des grands ensembles d'habitation de l'agglomération strasbourgeoise ", Géographie, économie, société, 13 (1), p. 69-92.

Grafmeyer, Y., Authier, J.-Y. (2008), Sociologie urbaine, Paris, Armand Colin, coll. « 128 ».

55. Rapport présenté au Congrès de I'Union Sociale pour l'Habitat, "Crise économique, mutations sociales: les HLM au cœur de la réponse. Exploitation de l'Union Sociale pour l'Habitat de l'enquête Logement de I'INSEE de 2006 ", 2011. 
Greenacre, M.J., Blasius, J. (2006), Multiple Correspondence Analysis and Related Methods, BocaRaton (Fla.), Chapman \& Hall.

HouARD, N. (2012), " La genèse du droit au logement opposable ", Politiques sociales et familiales, 107, p. 41-52.

ISRAEL, I. (2009), « Un droit de gauche? Rénovation des pratiques professionnelles et nouvelles formes de militantisme des juristes engagés dans les années 1970 », Sociétés contemporaines, 73, p . 47-72.

LAFORE, R. (1998), "Les nouveaux modes de régulation juridique ", dans Auvergnon, P., Martin, P., Rozenblatt, P., Tallard, M. (dir.), L'État à l'épreuve du social, Paris, Syllepse, p. 40-53.

LAscoumes, P., Serverin, É. (1986), Théories et pratiques de l'effectivité du droit, Droit et Société, 2, p. 127-150.

LeLEVRieR, C. (2010), "La mixité dans la rénovation urbaine : dispersion ou re-concentration ", Espaces et Sociétés, 140-141, p. 59-74.

Morel Journel, C., Sala Pala, V. (2011), « Le peuplement, catégorie montante des politiques urbaines néolibérales?", Métropoles [http://metropoles. revues.org/4536].

PeChu, C. (2006), Droit au Logement, genèse et sociologie d'une mobilisation, Paris, Dalloz, coll. " Nouvelle Bibliothèque de Thèses ».

Poupeau, F., TISSOT, S. (2005), « La spatialisation des problèmes sociaux", Actes de la recherche en sciences sociales, 159, p. 4-9.
Preteceille, E. (2006), "La ségrégation sociale a-t-elle augmenté ? La métropole parisienne entre polarisation et mixité ", Sociétés contemporaines, 62, p. 69-93.

SABBAGH, D. (2003), L'Égalité par le droit: les paradoxes de la discrimination positive aux États-Unis, Paris, Économica, coll. «Études politiques».

SAla PaLA, V. (2006), "La politique du logement social au risque du client? Attributions de logements sociaux, construction sociale des clients et discriminations ethniques en France et en GrandeBretagne ", Politiques et management public, 24 (3), p. 77-92.

SIMON, P. (2003), "Le logement social en France et la gestion des "populations à risques" ", Hommes et Migrations, 1246, p. 76-93.

Tissot, S. (2005), "Une discrimination "informelle" ? Usage de la notion de mixité sociale dans la gestion des attributions de logement social ", Actes de la recherche en sciences sociales, 159, p. 55-69.

VAN SAN, M. (2004), "Des quartiers de mauvaise réputation ", Déviance et Société, 28 (2), p. 211-231.

WARIN, P. (1999), "Les "ressortissants" dans les analyses des politiques publiques ", Revue française de science politique, 49 (1), p. 103-121.

WEILL, P.-E. (2012), "Savoir faire valoir son droit. Compétence statutaire et obtention d'un statut de "prioritaire" ", Sociologies pratiques, 24, p. 93-105.

WILson, W.J. (1997), When Work Disappears. The World of the New Urban Poor, New York (N. Y.), Vintage Books. 